\title{
NGO-Kommunikation 2.0 - Ist „KONY 2012“ der neue Maßstab?
}

\author{
Kerstin Plehwe, Sarah Bastgen
}

\begin{abstract}
Kernaussagen
Social Media sind für NGOs ein effektives Instrument der Wissensgenerierung, zur Mobilisierung und Bindung von Unterstützern sowie der internen Organisation. So lässt sich in den letzten Jahren eine stete Zunahme in der Nutzung der Social Media Angebote sowie eine verstärkte Integration der Anwendungen in die Kommunikationsmaßnahmen von NGOs beobachten. Anhand von Best Practice Beispielen werden hier die Möglichkeiten sowie daran anschließend die Herausforderungen der Social Media aufgezeigt. Diese dürfen bei aller Euphorie nicht vernachlässigt werden. Auch stellt sich nach der erfolgreichen Kampagne „KONY 2012“ die Frage, ob diese wirklich die richtige Messlatte des Social Media Einsatzes von NGOs sein kann. Für viele NGOs liegt der Fokus erst einmal auf dem koordinierten Aufbau eines Social Media Angebots und einer integrierten Kommunikationsstrategie verbunden mit der Schaffung eines Bewusstseins für die Möglichkeiten der neuen Kommunikationsformen.
\end{abstract}

\section{Zivilgesellschaft und Social Media - eine naturwüchsige Nähe}

Am Montag, dem 5.3.2012, wurde auf der Videoplattform Youtube ein 30- minütiges Video mit dem Titel „KONY 2012 “ veröffentlicht. Innerhalb von 48 Stunden konnte das Video 5 Millionen Klicks verzeichnen, nach 5 Tagen wurde es ganze 65 Millionen Mal aufgerufen (Kühl, 2012). Inhalt des Videos war eine Aufforderung zur Festnahme des ugandischen Rebellenführers Joseph Kony, der das Land zwanzig Jahre mit Entführungen und Terror in Atem gehalten hatte. Das Video war Auftakt einer koordinierten Online-Kampagne der NGO „Invisible Children“. So wurden gleichzeitig Facebook, Twitter, Youtube und ein Blog eingesetzt, um die Festnahme Joseph Konys zu erreichen und öffentlichen Druck auf die amerikanische Regierung auszuüben, ihre nach Uganda entsandten Militärberater dort zu belassen. Letztere hatten den Auftrag, die dortige Armee bei der Bekämpfung der Rebellen zu unterstützen.

Die Kampagne und auch die Organisation „Invisible Children“ geriet im Folgenden heftig in die Kritik, aufgrund von Ungenauigkeiten bei der Spendenverwendung und einer teilweise unsachlichen Kampagnenform. Zum einen wurde der Kampagne eine einseitige Wahrnehmung und Interpretation der Situation in Uganda vorgeworfen; zum anderen sei das Video emotional manipulativ, etwa durch die aktive Ein- beziehung des kleinen Sohns des Filmemachers. Ungeachtet dessen oder vielleicht gerade deswegen: Die Kampagne war ein unglaublicher Erfolg. Innerhalb kürzester Zeit hatten Menschen weltweit - u.a. gefördert durch massenmediale Berichterstattung - das Thema wahrgenommen. Die NGO hat es geschafft, Social Media ${ }^{1}$ so einzusetzen, dass ihre Botschaft den Globus umspannte.

Ein eindrucksvolles Beispiel wie Social Media von NGOs genutzt werden kann. Youtube, Facebook und Co. konnten hier ihre Wirkungskraft unter Beweis stellen. Im Blog der Süddeutschen Zeitung hieß es gar: „An ,Kony 2012' werden sich NGOs also in Zukunft messen lassen müssen." (Hofman, 2012)

Kommunikation ist für NGOs essentiell. Unabhängig davon, ob sie sich der Hilfe für Menschen verschrieben haben bzw. die verschiedensten Arten sozialer Dienstleistungen erbringen oder darauf abzielen, politische Entscheidungen zu Gunsten einer spezifischen Problemlage $\mathrm{zu}$ beeinflussen. NGOs klären die Öffentlichkeit auf, mobilisieren und organisieren die Bürger und betreiben Lobbying, um ihre Anliegen voranzutreiben und Druck auf Entscheidungsträger auszuüben. Die Potentiale von und damit auch die Erwartungen an Social Media in Hinblick auf die Kommunikation von NGOs sind groß: Die Kommunikation soll interaktiver, authentischer, symmetrischer, vernetzter und transparenter werden (vgl. Schultz \& Wehmeier, 2010, S. 421). Sie ermöglichen es, mit geringen Mitteln große Aufmerksamkeit zu erzeugen, potentielle Unterstützer zu generieren und eignen sich dementsprechend hervorragend als Fundraising-Instrument (vgl. Lubasch et al., 2010).

Diese Charakteristika machen die Anwendungen des Social Web zu einem beachtenswerten Instrument für zivilgesellschaftliche Organisationen wie NGOs. Alexandra Härtel und Serge Embacher (2011, S. 8) sehen gar einige Parallelen zwischen den Optionen der Social Media und dem System der Zivilgesellschaft an sich. Ihrer Meinung nach sind sowohl die (funktionierende) Zivilgesellschaft als auch Social Media von einem deliberativen Charakter geprägt. Ebenso sind Eigenschaften wie Toleranz, Partizipation und Reziprozität essen-

1 Der Begriff Social Media steht für eine interaktive und vernetzte Online-Kommunikation. (Lubasch et al, 2010). Dabei stehen verschiedene Formen der Social Media zur Verfügung. Man unterscheidet gewöhnlich zwischen 5 verschiedenen Anwendung der Social Media (vgl. Kilian \& Hass \& Walsh, 2011): Business Communities (z.B. Xing), Social Communities, (z.B. Facebook, MeinVZ), Weblogs (Bloganbieter, aber auch Microblogging Twitter), Knowledge Communities (z.B. wikipedia) und File Share Communities (z.B. MyVideo, Youtube). 
tiell für die vitale Ausprägung beider Systeme. Damit haben sie gewissermaßen eine „naturwüchsige“ Nähe zueinander.

\section{Vorteile und Potentiale der Social Media}

Die Vorteile der Social Media liegen auf der Hand: Ihre Nutzung ist relativ einfach und ohne viele technische Vorkenntnisse möglich. Auch können sich zum Teil erhebliche Kostenersparnisse im Vergleich zu Flyern, Buttons oder Werbestände auf offener Straße ergeben. Diese müssen allerdings gegen die Kosten für die professionelle Betreuung des Social Media Auftritts aufgerechnet werden, sodass hier immer der Einzelfall geprüft werden muss. Der entscheidende Vorteil liegt jedoch darin, dass mit einfachen Mitteln eine Vielzahl von Personen erreicht werden kann. Bei richtiger Nutzung sind sie damit ein effektives Instrument des „Campaigning“, insbesondere auch zur kurzfristigen Mobilisierung von Unterstützern. Wenn eine NGO es schafft, durch den Einsatz crossmedialer Verknüpfungen virale Effekte zu erzeugen, ist die Kampagnenarbeit einfach. Je häufiger eine Nachricht, ein Aufruf gepostet wird, desto mehr Menschen werden erreicht und können die Nachricht wiederum an ihre Community weiterleiten. Dritte fungieren als Botschafter der NGO und verbreiten das Anliegen der Organisation, ohne dass diese sich noch viel einbringen muss. Der „Like-Button“ bei Facebook erfüllt genau diese Funktion. Das „gelikte“ taucht auf der eigenen Seite auf und ist für den gesamten Freundeskreis sichtbar. Dieses wird dann wieder "gelikt" und so weiter und so fort. Kein anderes Medium hat bis dato die Möglichkeit einen derart viralen Effekt entstehen zu lassen.

Die aus der hohen Reichweite resultierenden Vorteile für die Mobilisierung sind wiederum essentiell für die politische Einflussnahme der Organisationen. Die NGO kann zur Untermauerung und Legitimation ihrer Position bzw. Forderungen auf eine breite Unterstützerbasis verweisen und damit mehr öffentlichen Druck auf Entscheidungsträger aufbauen.

Das Maß politischer Einflussnahme ist aber nicht nur von den Möglichkeiten der Mobilisierung, denn vielmehr von den (damit verbundenen) Fähigkeiten des Agenda Settings abhängig. Auch hier sind Social Media inzwischen zu einem einflussreichen Instrument avanciert und bringen Themen auf die öffentliche und politische Tagesordnung, die ansonsten eventuell nicht aufgetaucht wären. Die Funktion der traditionellen Medien als Gatekeeper von Themen und Informationen wird zunehmend untergraben. Damit haben insbesondere kleinere, finanzschwächere oder auch offline bisher kaum vernetzte NGOs die Möglichkeit, ihre Anliegen in die öffentliche Diskussion zu bringen. Dabei erlaubt es die Geschwindigkeit des Social Web, sich als Meinungsführer in einem Thema zu etablieren.

Des Weiteren eignen sich Social Media, um die bestehende Fundraising-Strategie zu bereichern. Die Spendenakquise ist in den letzten Jahren nicht leichter geworden - es gibt eine Vielzahl an Initiativen und Projekten, die auf Spenden angewiesen sind. Auch wenn Social Media den Vorteil haben, viele Menschen erreichen zu können, so sind damit allein die benötigten finanziellen Mittel nicht gesichert. Die beste Ansprache beim Fundraising ist, wie auch der jüngste Charity Scope der GfK wieder einmal bestätigte, mit 28,6\% immer noch der persönlich adressierte Brief (2012, S. 16). Social Media können allerdings äußerst effektiv als indirektes Instrument im Vorfeld der Ansprache eingesetzt werden, um die Organisation und ihre Anliegen bekannt zu machen. Die interaktiven, dialogorientierten Optionen eignen sich zur frühzeitigen Bindung an die eigene NGO, die sich zwar nicht unmittelbar auszahlen muss (im monetären, wie im praktischen Sinne), auf die jedoch im Lauf der Zeit zurückgegriffen werden kann. Auch im Beziehungsmanagement können sie effektiv eingesetzt werden, um Unterstützer möglichst langfristig an die Organisation zu binden. Selbst wenn Online-Fundraising bis dato noch für viele NGOs zu den im Vergleich weniger erfolgreichen Strategien der Mittelbeschaffung zählt, könnte sich zukünftig durch den geschickten Einsatz von Social Media ein vielversprechender Kanal zur Spendenakquise auftun.

Eine unschätzbar große Chance von Social Media liegt in ihrem Potential zur Wissensgenerierung (vgl. Härtel \& Embacher, 2011, S. 14). Durch einen aktiven Austausch und die Einbindung Interessierter in die eigene Arbeit können NGOs das geteilte Wissen anderer für ihre Projekte nutzen. Ausgehend von der Idee der Schwarmintelligenz kann das Wissen Externer neue Ideen und Ansätze hervorbringen, die ohne deren Partizipation nicht entstanden wären.

Zuletzt ergibt die organisationsinterne Nutzung von Social Media zum Teil völlig neue Optionen, insbesondere für dezentral organisierte NGOs. Nicht nur im Hinblick auf die Koordination und Organisation einer Kampagne, sondern auch intern zur Bewältigung der alltäglichen Arbeitsprozesse.

\section{Zahlen, Daten, Fakten}

In Deutschland nutzen inzwischen $73 \%$ der Bevölkerung über 14 Jahre das Internet gelegentlich (ARD/ZDF-Onlinestudie, 2011). Davon wiederum greifen $42 \%$ der Internetnutzer auf die Angebote der Social Communities zurück, was einer Steigerung von $17 \%$ in den letzten drei Jahren entspricht. Einzig Videoportale (58\%) und Knowledge Communities, insbesondere Wikipedia, $(70 \%)$ werden von noch mehr Personen frequentiert. Die Nutzung von regulären Weblogs, z.B. über Bloganbieter, aber auch das Microblogging ist mit $7 \%$ bzw. $3 \%$ unter den deutschen Internetnutzer weniger verbreitet als andere Social Media Angebote. Der Trend scheint aber auch hier ein weiteres Wachstum zu sein.

Wenig überraschend dürfte sein, dass ein Großteil insbesondere der Social-Networks-Nutzer eher jung ist. So sind $44 \%$ der Facebook-Nutzer unter 24 Jahren. Der mit 36\% größte Teil der Twitter-Nutzer ist zwischen 24 und 34 Jahre alt, nur $11 \%$ sind unter 24 Jahren. Auf das Videoportal Youtube wiederum greifen mit 53\% überwiegend 34 bis 54Jährige zu (Hartung, 2011).

Parallel zu den steigenden Nutzerzahlen lässt sich eine verstärkte Integration von Social Media in die Kommunikations- 
strategien der NGOs beobachten. Für die Studie „NGOs im Social Web“ werden seit 2009 jährlich 60 deutsche Organisationen aus den Bereichen Umwelt- und Naturschutz, Internationales sowie Soziales befragt (Kiefer 2009: 4). Während zum ersten Erhebungszeitpunkt lediglich 55 \% der NGOs auf ein Social Media Angebot wie Facebook, Twitter oder Youtube verweisen konnte, steigerte sich der Anteil bis 2011 um eindrucksvolle $33 \%$ (Kiefer, 2012). Gleichzeitig nahm die Anzahl der genutzten Anwendung pro NGO kontinuierlich zu. Die Anbieter Youtube und Facebook sind 2011 mit $73 \%$ bzw. $71 \%$ führend. Auch getwittert wird inzwischen von $63 \%$ der befragten Organisationen. Die klassischen Weblogs nutzen 2011 dagegen nur $35 \%$. Auffallend ist, dass insbesondere Facebook seit 2009 einen echten Boom erlebt hat; es erfolgte ein Sprung von 18 auf $71 \%$. YouTube und Twitter konnten ein ähnliches Wachstum verzeichnen (33 auf $73 \%$ bzw. 23 auf $63 \%$ ). Zudem erhöhte sich die Zahl der Unterstützer (Fans) der Facebook-Profile von durchschnittlich 174 auf 7652 - ein über 40-facher Anstieg. Hierbei muss allerdings berücksichtigt werden, dass sich die Zahl der Facebook-Nutzer in Deutschland in dieser Zeit verfünffachte (Kiefer, 2012).

\begin{tabular}{|l|c|c|}
\hline Organisation & Facebook-Fans & $\begin{array}{c}\text { Twitter-Follo- } \\
\text { wer }\end{array}$ \\
\hline Amnesty International (D) & 31.761 & 6.651 \\
\hline Ärzte ohne Grenzen & 59.429 & 4.861 \\
\hline ATTAC Deutschland & 13.825 & 6.024 \\
\hline BUND & 5.609 & 5.522 \\
\hline Deutsches Rotes Kreuz & 34.069 & 2.442 \\
\hline Foodwatch & 53.912 & 13.404 \\
\hline Greenpeace Deutschland & 65.471 & 46.534 \\
\hline Mehr Demokratie e.V. & 6.594 & 1.576 \\
\hline Oxfam (D) & 7.865 & 4.442 \\
\hline PETA Deutschland & 101.901 & 8.452 \\
\hline SOS Kinderdörfer & 10.601 & 1.496 \\
\hline WWF Deutschland & 96.928 & 13.785 \\
\hline Anzahl der Fans und Follower bekannter deutscher NGOs im Social Web \\
\hline
\end{tabular}

Quelle: Eigene Darstellung, Stand: 16.5.2012

Im Vergleich zu nordamerikanischen NGOs hinken deutsche, trotz dieses immensen Wachstums, immer noch ein wenig hinterher. Laut des aktuellen Annual Nonprofit Social Network Benchmark Report $(2012,12)$ nutzten in den USA $92 \%$ der 3522 in NGOs tätigen Befragten im letzten Jahr Social Media; 2012 sollen es $93 \%$ sein. Von diesen gebrauchen $89 \%$ Facebook für ihre Arbeit; in diesem Jahr sollen es unglaubliche $98 \%$ sein. $^{2}$

\section{Best Practice-Beispiele: Einsatz mit Strategie}

Die Statistiken deuten auf eine steigende Bedeutung von Social Media für die NGO-Kommunikation hin. Allerdings ist die reine Präsenz in den sozialen Netzwerken oder die Existenz eines Facebook-Accounts nicht ausreichend für den Erfolg. Hier gilt: Neben der Quantität bzw. der Frequenz der Updates zählen vor allem qualitative Aspekte. Entscheidend ist die Social Media-Strategie: Nicht dass es genutzt wird, sondern wie!

Als Vorreiter im Bereich Social Media gelten die deutschen Subsektionen von Greenpeace und des WWF. Greenpeace ist aktiv in vier Social Communities; mit Twitter, Youtube, zwei File Share Communities und in einem eigenen Blog zeigen sie maximale Präsenz. Durch systematische Verlinkungen auf die verschiedenen Anwendungen sind die Inhalte überall und jederzeit verfügbar. So wird nicht nur kontinuierlich auf laufende und zukünftige Kampagnen hingewiesen, sondern auch über vergangene Aktionen berichtet. Durch Filme auf dem eigenen Youtube-Kanal können Interessierte für sie relevante und spannende Aktionen nachverfolgen. Diese Strategie kommt bei den Zielgruppen an. Fast 62000 Facebook-Fans bei einem deutschen Durchschnitt von 7600 sprechen für sich. ${ }^{3}$ Bei Youtube hat der Greenpeace-Kanal 5000 Abonnenten bei einem Durchschnitt von 335 und auch die ca. 44.000 Follower bei Twitter liegen weit über dem Durchschnitt.

Was Greenpeace Deutschland jedoch zu einem wirklichen Vorreiter macht ist das eigene Soziale Netzwerk: GreenAction; eine offene Mitmach-Plattform für Organisationen, Initiativen und Einzelpersonen. Mittels dieser Plattform kann jeder selbst aktiv werden; eigene Kampagnen schalten, andere Aktionen unterstützen und sich mit Umweltaktiven vernetzen. Eingerahmt wird GreenAction durch einen Blog, Twitter und einen Facebook-Auftritt. Momentan sind bei GreenAction ca. 12 000 User registriert, über 4000 folgen der Plattform bei Twitter (Stand: 30.5.2012). GreenAction ist inzwischen Geburtsort vieler Kampagnen unter dem Dach von Greenpeace Deutschland. Dabei reicht die Bandbreite von regionalen Tierschutzprojekten bis zu Kampagnen gegen das Auftreten von Coca Cola in Indien.

Auch dem WWF Deutschland wird Vorbildfunktion im Bereich Social Media zugesprochen. Mit fast 100.000 Facebook-Fans ist der WWF die erfolgreichste deutsche NGO bei Facebook. Sein sehr abgestimmtes Angebot im Social Web lässt sich zudem an ca. 15.000 Twitter-Followern ablesen (Zahlen vom 6.6.2012). Der WWF hat sich schon relativ früh mit der Entwicklung einer Social Media Strategie beschäftigt und gehörte zu den ersten Organisationen, die Facebook und Co. bewusst und geplant für ihre Kampagnen und Ziele einsetzten. So hat der WWF 2010 als erste deutsche NGO die Möglichkeit der Facebook-Petition erfolgreich genutzt.

Eine sehr erfolgreiche, primär Social Media basierte Kampagne unter dem Titel „Free Giacobbo“ gelang Amnesty Schweiz im Mai 2011. Auf Youtube tauchte ein Video auf, in dem der bekannte Schweizer Satiriker Viktor Giacobbo behauptete, er sei wegen eines Witzes über den Schweizer Bun-

2 Der Annual Nonprofit Social Network Benchmark Report zeigt auch deutlich, dass für den nordamerikanischen Raum schon deutlich ausgereifteres Datenmaterial erhoben wird und die Forschung zum Thema Social Media im Allgemeinen, aber auch Social Media für NGOs, einen größeren Stellenwert einnimmt, als dies bis dato in Deutschland der Fall ist.

3 Durchschnittszahlen bei Kiefer 2012. Nutzerzahlen vom 14.5.2012. 
desrat verhaftet worden. Bei Twitter und Facebook wurde diese Nachricht sofort aufgenommen und verbreitet. Am nächsten Tag berichteten die Online-Ausgaben der großen Printmedien darüber.

Natürlich war Giacobbo nicht verhaftet worden; vielmehr wollte er auf die Verhaftung seines Kollegen Zarganar aus Burma aufmerksam machen. Ziel der Aktion war, möglichst viele Unterschriften für dessen Freilassung zu sammeln. Amnesty hatte das Video produziert und gezielt 50 äußerst aktive Twitter-Nutzer angeschrieben. Zudem erhielten am darauffolgenden Tag 20.000 Amnesty-Unterstützer per EMail Informationen über die angebliche Verhaftung Giacobbos, mittels derer sie zur Petition für Zarganar geleitet wurden. Die traditionellen Medien wurden vorher nicht über die Aktion informiert. Ebenso wurde Facebook nicht aktiv durch die Amnesty eingebunden, allerdings wurde nach eigenen Aussagen auf den viralen Effekt vertraut.

Die Kampagne war dank eben dieses Effekts ein voller Erfolg. Innerhalb von drei Tagen konnten online 14.000 Unterschriften gesammelt werden. ${ }^{4}$ Gleichzeitig hatte Amnesty es aber auch durch die erhöhte Aktivität im Netz geschafft, die Aufmerksamkeit der großen Zeitungen zu erregen und sich diese zunutze zu machen ohne selbst direkt an sie heranzutreten. Damit ist der NGO der für effektive Einflussnahme so wichtige Spagat geglückt: Die Möglichkeiten der Social Media optimal für die eigenen Zwecke einzusetzen und gleichzeitig crossmedial zu wirken.

Die Evaluation dieser Kampagne fällt nicht nur aus kommunikationsstrategischen Gesichtspunkten positiv aus: Zarganar wurde Ende 2011 freigelassen.
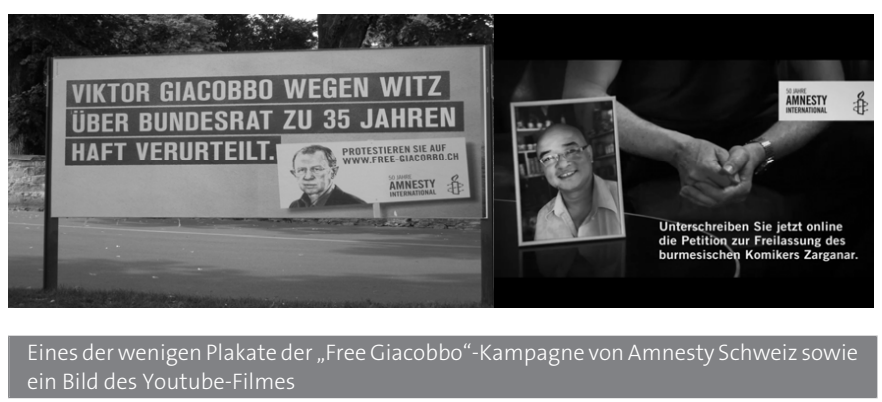

Ein weiteres Beispiel für den gelungenen externen wie internen Einsatz von Social Media ist die Entwicklungshilfeorganisation „2aid.org“. Nach eigenen Angaben werden zahlreiche Social Media-Tools für organisationsinterne Abläufe genutzt (2aid.org, 2012), wodurch sich erhebliche Verwaltungskostenersparnisse ergeben. Die NGO selbst hat kein zentrales, gemeinsames Büro - das Kernteam ist über das gesamte Land verteilt. Auch die Aktionen der NGO finden überwiegend online statt. So ist es sehr leicht, Menschen an unterschiedlichen Orten zu mobilisieren und mit einzubeziehen, was dazu führt, dass der Kreis der aktiven Unterstützer räumlich sehr offen gehalten werden kann. Für 2aid.org erweisen sich Social Media als ein echtes Instrument zur Kostensenkung; nur damit kann der Versuch gewagt werden, die Spen- den zu $100 \%$ in Projekte vor Ort fließen zu lassen. Auch im Fundraising ist die NGO ein Vorreiter: Ein Großteil der Spenden wird Online eingeworben und die Unterstützer können auf Facebook und im Blog die unmittelbare Verwendung ihrer Spende verfolgen.

Alle diese Beispiele zeigen, dass für eine erfolgreiche Nutzung von Social Media, die die jeweilige Zielgruppe erreicht, nicht allein die bloße Existenz im Netz wichtig ist. Entscheidend ist häufig die Existenz einer expliziten Social MediaStrategie. Greenpeace, WWF und Amnesty Schweiz stellen hier Kampagnenkoordination und Information in den Mittelpunkt, um in kurzer Zeit Unterstützer - etwa bei einer Unterschriftenaktion - akquirieren zu können. Möglich sind aber auch andere Strategien und daraus resultierende Einsatzgebiete: Fundraising, Identifikation von Trends, aktive Einbeziehung der Unterstützer und Mitglieder in die Positionsfindung oder Suche nach Ehrenamtlichen. ${ }^{5}$ Auch können die Kanäle, insbesondere die Social Communities, für klassisches Beziehungsmanagement genutzt werden, um mit der NGO „in-touch“ zu bleiben. Häufig findet man bei genauerer Betrachtung eine (bestenfalls) abgestimmte Mischung der verschiedenen Strategien. Entscheidend ist, dass bei der Strategiewahl die Frage im Zentrum steht, welches Ziel das eigene Social Media-Auftreten verfolgen soll.

Die hier dargelegten Best-Practice Beispiele haben ihre Ziele und ihre Social Media- Strategie klar definiert. Demgegenüber stehen jedoch immer noch zahlreiche Organisationen ohne ausdifferenzierte Social Media-Strategie. Häufig werden die Potentiale des Social Web weniger für die Kampagnenarbeit, denn lediglich zur Verbreitung allgemeiner Informationen genutzt. Zudem wird oft nur unregelmäßig über diesen Kanal kommuniziert. Andere nehmen nur eine unzureichende Verlinkung und Integration mit anderen Kommunikationsangeboten vor oder verzichten ganz und gar auf die Nutzung von Social Media. Der Verzicht auf Social Media oder aber der optimierungsbedürftige Einsatz resultieren aus den, zum Teil erheblichen, Anforderungen an die Organisationen selbst, die sich aus den oben erläuterten Potentialen und Vorteilen ergeben. Viele NGOs sehen sich immer wieder mit tatsächlichen oder auch nur subjektiv wahrgenommenen Nachteilen, Herausforderungen und Ängsten konfrontiert.

\section{Warum bleiben einige außen vor? - Herausforderungen und Grenzen von Social Media}

Gute Kommunikation orientiert sich immer an den Zielgruppen. Und genau aus diesem Grund stellt sich bei einigen NGOs gar nicht erst die Frage der Notwendigkeit eines Social Media Auftritts, da deren Zielgruppen, beispielsweise aufgrund genereller Vorbehalte gegenüber dieser Art der Kommunikation, das Social Web nicht zu ihren präferierten Kommunikationskanälen zählen.

4 Übergeben wurden der Botschaft Burmas letztlich 25.000 Unterschriften.

5 4th Annual Nonprofit Social Network Benchmark Report, 2012, S. 16 sowie Kiefer, 2012. 
Bei vielen anderen Organisationen ist jedoch ein Zustand der (kritischen) Zurückhaltung gegenüber der Kultur der Sozialen Medien zu beobachten der von Hannes Jähnert (Jähnert, 2012) mit dem Begriff Kulturschock umschrieben wird. Die Überwindung dieses Zustands erfordert eine gewisse Zeit und das Erlernen entsprechender Kompetenzen. Dabei können Maßnahmen der Unterstützung durch bereits Aktive ihren Beitrag leisten.

Generell erfordert die effektive und erfolgreiche Nutzung von Social Media von vielen NGOs ein Umdenken. Grundvoraussetzung dafür ist, dass NGOs Social Media nicht nur als Werbemittel nutzen, sondern in ihnen eine Dialogplattform sehen. Dafür ist eine Abkehr vom Prinzip der „alten Kommunikation in neuen Kanälen“ hin zu einem partizipativen Miteinander von Organisation und Mitgestalter im Social Web nötig (vgl. Kiefer, 2012). NGOs müssen, wenn sie diesen Vorteil nutzen wollen, ihr Gegenüber im Netz ernst nehmen und dessen Vorschläge, Ideen und Beiträge zumindest einer gewissenhaften Betrachtung unterziehen. Andernfalls droht die Mitarbeit des anderen wieder zu verflachen und potentielles Wissen oder Ideen können nicht zum Tragen kommen. Der Herausforderung dieser neuen Kommunikationsform im Social Web sind noch nicht alle NGOs gewachsen oder tun sich aufgrund der vorherrschenden Organisationskultur schwer mit ihr.

Ferner bergen die dem Social Web inhärenten Optionen des Dialogs mit Interessierten auch immer das Risiko des Kontrollverlustes über Debatten. Sobald viele Akteure involviert sind, hat die Organisation weniger Einfluss auf Verlauf, Inhalt und Ausgang einer Diskussion - sie riskiert den Verlust ihrer Deutungshoheit. Damit einher geht, dass Pläne und Vorhaben durch Einwände aus der später hinzu geschalteten Webgemeinde, die häufig nicht alle Einflussfaktoren kennen (können), zerredet und verworfen werden. Die Angst über einen möglichen Kontrollverlust über die Kommunikation teilen viele Organisationen. In der Studie „Social Media Governance“ wurden 596 Kommunikationsmanager und PRVerantwortliche in Unternehmen, Behörden und NPOs zum Einsatz von Social Media befragt: 55 \% sehen ein Risiko im schlecht steuerbaren bzw. unkontrollierbaren Kommunikationsverlauf und damit die Gefahr des Verlustes der Kontrolle über die Selbstdarstellung der Organisation (vgl. Fink \& Zerfaß, 2011, S. 22).

Der Verlust der Kontrolle und die Konsequenzen dieses Verlusts werden inzwischen auch mit dem Begriff „Shitstorm“ bezeichnet. Gemeint ist damit die Empörungswelle, „,bei de[r] massenhafte öffentliche Entrüstung, sachliche Kritik sowie auch völlig unsachliche Beiträge das Internet zu einem Thema überfluten “ (King, 2012). In der Regel fällt es der betroffenen Organisation schwer, die Flut der Kommentare und Anfragen zeitnah zu bearbeiten und der Diskussion Herr zu werden. Einem Shitstorm sah sich am 22.6.2011 auch der WWF Deutschland ausgesetzt. Bei der bis dato führenden NGO im Bereich Social Media in Deutschland brach im Nachgang einer kritischen WDR-Dokumentation unter dem Titel „Der Pakt mit dem Panda - Was uns der WWF verschweigt" eine Welle der Empörung bei Facebook, Twitter und auf der Internetseite über den WWF herein. Nach eigener Aussage waren die Verantwortlichen bei WWF zunächst personell, technisch, aber auch inhaltlich mit den Geschehnissen überfordert (vgl. Hannemann, 2011). Es dauerte einen Tag (im Netz ein langer Zeitraum), bis der WWF sowohl auf inhaltlicher als auch auf technischer Ebene Möglichkeiten der Krisenkommunikation bzw. der Diskussion und Gegendarstellung entwickeln konnte. Die Masse der Kommentare war nicht immer abzuarbeiten. Der WWF hat seine Lehren aus diesen Ereignissen gezogen und ist mit seinem Krisenmanagement im Social Web im Anschluss an die Geschehnisse selbstkritisch umgegangen und das nicht nur intern, sondern auch öffentlich. Die zentrale Botschaft der Social Media Managerin - die u.a. zu eben diesem Thema auf der diesjährigen re:campaign vertreten war ist, dass man sich auf einen Shitstorm nicht nur vorbereiten kann, sondern auch muss; er ist nur mit entsprechender Vorbereitung und richtigem Ressourceneinsatz zu bewältigen. Eines zeigt sich aber sehr deutlich: Niemand ist vor einem Shitstorm geschützt - auch NGOs nicht.

Auch wenn nicht jeder Kontrollverlust über die Selbstdarstellung und die Diskussion so heftig ausfallen wie für den WWF, so ist die Angst davor zumindest einer der Gründe, warum gelegentlich Zurückhaltung bei Umfang und der Art und Weise des Einsatzes von Social Media an den Tag gelegt wird.

Ein weiterer Grund ist der notwendige Zeitaufwand bei effektivem und koordiniertem Einsatz. Zwar stehen auf der einen Seite Kostenersparnisse, auf der anderen Seite sollte man den Ressourcenaufwand keinesfalls unterschätzen. Die Professionalisierung des Social Media-Engagements erfordert Personal und Zeit, denn abhängig vom Umfang der Online-Nutzung setzt der intensive, koordinierte Einsatz Fachkenntnisse in Online-PR voraus und insbesondere die Implementierung einer Social Media-Policy ist nicht nur aufwendig, sondern bedarf entsprechender Expertise. Die Mehrheit der großen NGOs haben inzwischen für Social Media-Auftritte qualifizierte Social Media- oder Community-Manager (vgl. Kiefer, 2012). Für kleinere NGOs oder Vereine stellt dieser erforderliche Ressourceneinsatz nach wie vor häufig ein Problem dar. Entweder sind die erforderlichen Mittel nicht vorhanden und/ oder es mangelt an Fachwissen (vgl. Reiser, 2012).

Eine weitere nicht zu vernachlässigende Herausforderung ist die der Evaluation. Öffentlichkeits- und Kampagnenarbeit ist grundsätzlich mit der Problematik aussagekräftiger Kriterien der Erfolgsmessung belastet. Bezüglich Social Media wird diese Situation noch verstärkt, da die Vernetzung nicht nur der unterschiedlichen Anwendungen, sondern auch der Social Media mit anderen Kommunikationsinstrumenten die Rückführung auf einen bestimmten Kanal nahezu unmöglich macht. Gerade in Hinblick auf das Beziehungsmanagement wird die Evaluation der Maßnahmen, die zu einer Verbindung geführt haben, immer schwieriger. So verlassen sich auch $63 \%$ der im Annual Nonprofit Social Network Benchmark 
Report Befragten eher auf „weiche“ Kriterien zur Messung des Return on Investment ihrer Social Media Nutzung. $32 \%$ versuchen sich erst gar nicht in der Evaluation und nur $5 \%$ geben an, finanzielle Messungen im Rahmen der Evaluation vorzunehmen (2012, S. 17).

\section{Schlussgedanken}

Social Media haben ihre Wirkungskraft bewiesen. Nicht nur das Beispiel „Kony 2012“, sondern auch die so genannten „Facebook-Revolutionen“ im arabischen Raum haben gezeigt, dass die Zivilgesellschaft das Social Web effektiv für sich nutzen kann.

NGOs haben hier ein weiteres Instrument zur Ansprache potentieller Unterstützer; verbunden mit der Möglichkeit, mit diesen in die direkte und relativ unkomplizierte Kommunikation zu treten. Bei allen Vorteilen, welche die Kanäle bieten, dürfen Grenzen und Herausforderungen aber nicht aus dem Blickfeld geraten. Social Media sollten nicht als die neuen Heilsbringer verstanden werden. Vielmehr sind sie eine Ergänzung und Erweiterung der bisherigen Möglichkeiten der Kommunikation und sollten als Teil einer integrierten Dialogkommunikationsstrategie verstanden werden.

Für NGOs besteht des Weiteren bei Überschätzung des Mediums die Gefahr, dass ihre Unterstützer in einen „Slacktivism“ verfallen: Engagement kann man mit einem Mausklick zeigen. Es ist relativ einfach und unverbindlich. Es ist faul und aktionistisch zugleich. Allerdings sind die Organisationen zum großen Teil auf mehr und vor allem offline stattfindende Beteiligung angewiesen. Es kommt also darauf an, das digitale auch in Offline-Engagement umzuwandeln. Dabei erweisen sich neben Mitmachangeboten in der „realen“ Welt crossmediale Verknüpfungen als ein Schlüssel zum Erfolg. Zum einen sollten mehrere zielgruppenspezifische Medienkanäle angeboten werden, um Nutzer zu erreichen oder diesen die Option zu bieten, sich interaktiv mit dem Thema oder der Organisation zu beschäftigen. Zum anderen sollten die gewählten Kommunikationskanäle inhaltlich, formal und zeitlich integriert sein, um die gewünschten Synergieeffekte hervorzurufen.

In Hinblick auf zukünftige Trends und Entwicklungen sei der Blick an dieser Stelle auf die USA gerichtet. Dort waren NGOs bereits 2009 in Hinblick auf ihre Social Media-Strategie da, wo die meisten deutschen Organisationen sich heute befinden. Allerdings ist fraglich, ob hier eine ähnliche Entwicklung abzusehen ist. $\mathrm{Zu}$ unterschiedlich sind (noch) die Techniknutzungskulturen. Ebenso können professionalisiertes Fundraising und Freiwilligenmanagement in den USA auf eine deutlich längere Tradition zurückblicken. Zudem muss das unterschiedliche Staatsverständnis und die weniger ausgeprägt Individualisierung in Deutschland berücksichtigt werden (vgl. Initiative ProDialog, 2011, S. 16; 2010, S. 6). Dennoch ist eine stärker werdende Professionalisierung dieser Bereiche auch in Deutschland zu beobachten. Die Zunahme mobiler Kommunikationsmittel erlaubt neue Strategien wie etwa SMS erfolgreich einzusetzen. Auch wird sich das Nut- zungsverhalten weiter ändern. Daran muss sich wiederum die Strategiewahl von NGOs wieder anpassen.

Ohne Frage, Kampagnen wie „KONY 2012“ sind aufgrund ihrer hohen Reichweite ein Erfolg. Allerdings zeigt diese Kampagne auch zweierlei: Erstens bringen die große Reichweite und die Möglichkeit der viralen Verbreitung von Botschaften eine Verantwortung für den Inhalt der Botschaft mit sich. Die außerordentliche kritische Diskussion über „KONY 2012“ im Nachgang der Kampagne deckte Schwächen und Ungenauigkeiten auf. Diese konnten der breiten Öffentlichkeit nur schwerlich vermittelt werden, weil die Kampagne bereits im Vorfeld ihre große Wirkung entfaltet hatte. Zweitens muss in Frage gestellt werden, ob „KONY 2012“ wirklich die Messlatte für zukünftige NGO-Kampagnen sein sollte. Für viele NGOs steht erst einmal der koordinierte Aufbau eines Social Media-Angebots und einer Social Media-Strategie im Mittelpunkt. Den Globus umspannende Kampagnen können für viele NGOs nicht das vorrangige Ziel sein. Vielmehr wird es auf eine grundständige Arbeit im Bereich Social Media und die Schaffung eines Bewusstseins für die Möglichkeiten der neuen Kommunikationsformen ankommen.

\section{Literatur}

2aid.org (2012). Verfügbar unter www.2aid.org/inhalt/ueber_uns/ team.html [8.6.2012]

4th Annual Nonprofit Social Network Benchmark Report (2012). Verfügbar unter http://nonprofitsocialnetworksurvey.com/ [8.6.2012]

ARD/ZDF- Onlinestudie (2011). Verfügbar unter www.ard-zdf-onlinestudie.de/index.php?id=325 [8.6.2012]

Fink, S. \& Zerfaß, A. (2011): Social Media Governance 2010, Ergebnisse einer Studie bei Kommunikationsverantwortlichen in Unternehmen, Behörden, Verbänden und Non-Profit-Organisationen. Verfügbar unter www.socialmediagovernance.eu [8.6.2012]

GfK Charity Scope (2012). Verfügbar unter www.spendenrat.de/filearchive/51f5cc7df589a49c7a7e07dcc149b13 d.pdf [8.6.2012]

Härtel, A. \& Embacher, S. (2011). Internet und digitale Bürgergesellschaft - Neue Chancen für Beteiligung. Eine Studie des CCCD - Centrum für Corporate Citizenship Deutschland. Verfügbar unter www.cccdeutschland.org/sites/default/files/CCCDebatte08_Internet\%20und \%20digitale\%20Buergergesellschaft_2011.pdf [8.6.2012]

Hannemann, P (2011). Vortrag „Was tun wenn der Shitstorm kommt?“. Verfügbar unter www.ambuzzador.com/2011/10/25/buzzattack11paula-hannemann-vom-wwf-deutschland-und-ihre-10-shitstorm-tipps/ [8.6.2012]

Hartung, B. (2011). Social Media: Nutzerzablen im September 2011. Verfügbar unter http://birgerh.de/2011/09/30/social-media-nutzerzahlen-imseptember-2011/ [8.6.2012].

Hofman, N. (2012). Umstrittenes Video „KONY 2012“-Eine Kampagne, die frösteln lässt ". Verfügbar unter www.sueddeutsche.de/digital/umstrittenes-video-kony-eine-kampagne-die-froesteln-laesst-1.1305052 [8.6.2012]

Initiative ProDialog (2010). Message Money Mobilization, Blick in die USA: Erfolgreiche Non-Profit-Kommunikation. Eine Studie der Initiative ProDialog.

Initiative ProDialog (2011). Motivieren Mobilisieren Mitarbeiten, Freiwilligenmanagement in den USA: Best Practice für Deutschland. Eine Studie der Initiative ProDialog.

Jähnert, H. (2012). Kulturschock Social Web: Soziale Medien kennen und leben lernen. Verfügbar unter http://hannes-jaehnert.de/wordpress/ 2012/04/26/kulturschock-social-web-soziale-medien-kennen-und-lebenlernen/ [8.6.2012]

Kiefer, K. (2009). NGOs im Social Web Ein inhaltsanalytische Untersuchung zum Einsatz und Potential von Social Media für die Öffentlich- 
keitsarbeit von gemeinnützigen Organisationen. Verfügbar unter: http:// netzwerkpr.de/wp-content/uploads/2010/04/Kiefer_NGOs-im-SocialWeb.pdf [8.6.2012]

Kiefer, Kathrin (2012). NGOs im Social Web, Status Quo zum Social Media Einsatz in gemeinnützigen Organisationen. Verfügbar unter: http://netzwerkpr.de/index.php/2012/04/status-quo-zum-social-mediaeinsatz-in-npos/ [8.6.2012]

Kilian, T., Hass, B. \& Walsh, G. (2011). Grundlagen des Web 2.0. In Hass, B., Walsh, G. \& Kilian, T. (Hrsg.), Web 2.0 - Neue Perspektiven für Marketing und Medien, (3-20). Berlin: Springer.

King, H. (2012). Shitstorm. In Neues Wörterbuch der Szenesprachen. Verfügbar unter http://szenesprachenwiki.de/definition/shitstorm/ [8.6.2012]

Kühl, E. (2012). KONY 2012: Mit Social Media gegen den Völkermörder?. Verfügbar unter http://blog.zeit.de/netzfilmblog/2012/03/08/josephkony-2012-social-media-uganda-film/ [8.6.2012]

Lubasch, T., Böhm, F. \& Nöll, F. (2010). Social Media Report. Non-Profit-Organisationen starten in das Social Web. Spendino GmbH.

Reiser, B. (2012). Nonprofits müssen ins Internet und dieses mitgestalten. Blog noprofits-vernetzt.de. Verfügbar unter http://blog.nonprofitsvernetzt.de/index.php/nonprofits-mussen-ins-internet-und-dieses-mitgestalten/ [8.6.2012]

Schultz, F. \& Wehmeier, S.W. (2010). Online Relation. In: Schweiger, W. \& Beck K. (Hrsg.), Handbuch Online-Kommunikation (409-431). Wiesbaden: Verlag für Sozialwissenschaften.

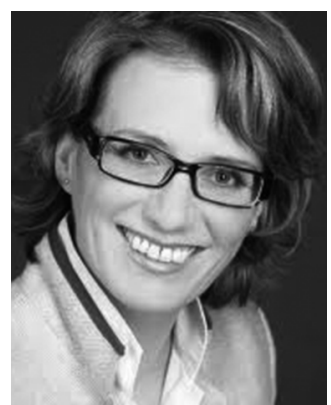

Kerstin Plehwe ist Vorsitzende der überparteilichen Initiative ProDialog Gesellschaft zur Förderung von Demokratie und Engagement $\mathrm{mbH}$ in Berlin. , gilt die Geschäftsführerin der Initiative ProDialog und Gründerin des Internationalen Instituts für Politik \& Gesellschaft als engagierte Protagonistin moderner Kommunikationswege und eines neuen Leadership-Verständnisses in Politik und Wirtschaft. E-Mail: plehwe@prodialog.org.

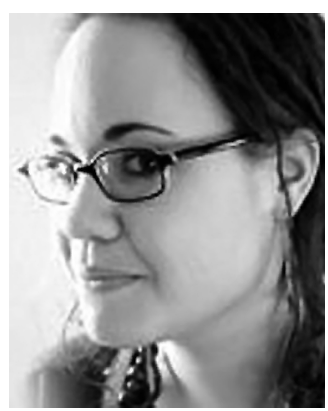

Sarah Bastgen ist Leiterin Politik und Gesellschaft der Initiative ProDialog und promoviert an der FU Berlin zum Thema Kommunikationsstrategien von NGOs im Europäischen Mehrebenensystem. Ihre Arbeitsschwerpunkte sind politische und zivilgesellschaftliche Kommunikation sowie europäische Zivilgesellschaft. E-Mail: bastgen@prodialog.org.

\title{
Die Transformation der öffentlichen Verwaltung durch die sozialen Medien
}

\author{
Boris von Chlebowski
}

\begin{abstract}
Kernaussagen
Mit Facebook, Twitter und Youtube haben die sozialen Medien die Art und Weise revolutioniert, wie Konsumenten und Bürger heutzutage Informationen sammeln, kommunizieren und zusammenarbeiten. Nachdem erfolgreiche Unternehmenskommunikation bereits seit längerem auf soziale Medien setzt, ist nun auch die öffentliche Verwaltung am Zug. Fest steht schon jetzt: Web 2.0 und die sozialen Medien werden die Arbeitsabläufe und Kultur der Verwaltung nachhaltig verändern.
\end{abstract}

\section{Revolutionen, Unternehmen und aktive Bürger}

\subsection{Die digitale Revolution}

Wenn man heute die Nachrichten verfolgt, ist nicht zu übersehen, welchen enormen Einfluss soziale Medien mittlerweile als Ausgangspunkt für Veränderungen haben. Die Menschen beziehen nicht nur ihre Nachrichten über soziale Medien, son- dern die sozialen Medien selbst stehen im Mittelpunkt der Nachrichten.

Nehmen wir zum Beispiel den Arabischen Frühling. In vielen Staaten Nordafrikas und des Nahen Ostens wurden die Regierungen ins Wanken gebracht - durch eine Massenbewegung, die größtenteils über soziale Medien initiiert und gelenkt wurde. Diese „E-Revolution“ hat sich im Laufe der Zeit über alle Grenzen hinweggesetzt, ob Klassen oder Geschlechter, politische oder nationale Territorien. Die Berichterstattung über die Ereignisse stützte sich in großen Teilen auf soziale Medien, die als Quelle für lokale Informationen dienten. So wurden aus persönlichen Schicksalen im Handumdrehen internationale Nachrichten.

Die öffentliche Verwaltung kann viel von den Ereignissen des Arabischen Frühlings, der Occupy-Bewegung oder aus „Stuttgart 21“ lernen. Jüngstes Beispiel ist das Anti-Produktpiraterie-Handelsabkommen ACTA, dessen spektakuläres Scheitern im Europäischen Parlament am 4. Juli 2012 wesentlich auf auf eine sorgfältig geplante Online- wie Offline-Kampagne zurückzuführen ist. Die Sozialen Medien und Web 2.0- 